\title{
Distribution of IgG4- and/or IgG-Positive Plasma Cells in Hashimoto's Thyroiditis: An Immunohistochemical Study
}

\author{
Masaru Kojima $^{a}$ Mitsuyoshi Hirokawa ${ }^{b}$ Haruji Kuma $^{b}$ Eijun Nishihara ${ }^{b}$ \\ Nobuhide Masawa ${ }^{a}$ Naoya Nakamurac ${ }^{c}$ Akira Miyauchib \\ a Department of Anatomic and Diagnostic Pathology, Dokkyo University School of Medicine, Mibu, \\ ${ }^{b}$ Center for Excellence in Thyroid Care and Department of Pathology, Kuma Hospital, Kobe, and \\ 'Department of Pathology, Tokai University School of Medicine, Isehara, Japan
}

\section{Key Words}

Hashimoto's thyroiditis • IgG4-related sclerosing disease •

Plasma cells $\cdot$ Immunohistochemistry

\begin{abstract}
Background and Study Aim: Recently, immunohistochemistry has shown numerous IgG4-positive plasma cells in a subset of Hashimoto's thyroiditis (HT), and this type of HT (IgG4 HT) appears to be a subtype of IgG4-related sclerosing disease. However, little is known about the distribution pattern of plasma cells in IgG4 thyroiditis. To clarify the distribution pattern of IgG4-positive plasma cells, 33 cases of HT demonstrating abundant lymphoplasmacytic infiltrate were studied. Methods: Using formalin-fixed paraffin-embedded sections, histological, immunohistochemistry and polymerase chain reaction were performed. Results: Fourteen cases were classified as IgG4 HT and 19 cases were non-IgG4 HT. Histologically, there was no significant difference between the 2 groups with regard to the degree of stromal fibrosis, lymphoid follicle formation, or the presence of phlebitis or fibrous thyroiditis. The present study demonstrated 2 distribution patterns of IgG4- and/or lgG-positive plasma cells, namely the interfollicular $(n=31)$ and intrafollicular + interfollicular patterns $(n=2)$. Interfollicular plasma cells
\end{abstract}

were always polytypic intracytoplasmic immunoglobulin in all 33 cases. However, intrafollicular plasma cells in 2 lesions had monotypic kappa light chain by immunohistochemistry. Conclusion: The present study demonstrated that the majority of IgG4 HT and non-lgG HT cases showed an interfollicular distribution pattern of lgG4- and/or lgG-positive plasma cells.

Copyright $\odot 2010$ S. Karger AG, Basel

\section{Introduction}

Hashimoto's thyroiditis (HT), which is characterized by the presence of goiter and serum thyroid autoantibodies, is the most common type of thyroiditis [1]. The diagnostic criteria for HT have been well described. However, HT exhibits various clinicopathological presentations and its pathogenesis is poorly understood [1]. IgG4-related sclerosing disease is a recently recognized entity, clinically characterized by mass-forming lesions in the exocrine glands, extranodal organs (most frequently pancreas, biliary tract, salivary glands and lacrimal gland) and

M.K. and M. H. contributed equally to this work.

\section{KARGER}

Fax +41613061234 E-Mail karger@karger.ch www.karger.com

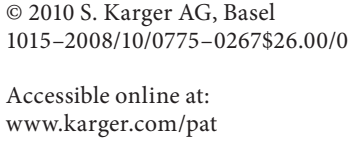

E-Mail k-masaru@dokkyomed.ac.jp 
lymph nodes as well as elevated serum IgG4 level, and shows a good response to steroid therapy $[2,3]$. It is characterized by prominent lymphoplasmacytic infiltration and sclerosis as well as numerous IgG4-positive plasma cell infiltrations [3]. The fibrous variant of HT is characterized by a marked fibrous replacement of one third or more of the thyroid parenchyma $[4,5]$. Harach and Williams [6] found that the fibrous variant of HT contained numerous IgG-positive plasma cells. In 2005, Komatsu et al. [7] demonstrated a high prevalence of hypothyroidism among patients with autoimmune pancreatitis. Histopathological and immunohistochemical findings of the fibrous variant of HT are similar to those of IgG4-related disease. Recently, Li et al. $[8,9]$ demonstrated that from both clinical and histopathological [i.e. presence of prominent fibrosis, numerous IgG4-positive plasma cells and elevated serum IgG4 level (>135 mg/dl)] perspectives, IgG4 HT and non-IgG4 HT appear as distinct entities [3]. They emphasized that measuring the serum IgG4 level provided a useful method of distinguishing IgG4 HT from non-IgG4 HT [9]. In IgG4-related lymphadenopathy, Sato et al. [10] classified two types of IgG4-related lymphadenopathy by the infiltration pattern of IgG4positive plasma cells: interfollicular plasmacytosis and intragerminal center plasmacytosis. However, little is known about the distribution pattern of IgG4-positive plasma cells in IgG4 HT. To clarify the presence or absence of 2 types of IgG4-positive plasma cell infiltration in IgG4 HT, we studied 33 cases of HT demonstrating prominent lymphoplasmacytic infiltration in comparison with non-IgG4 HT.

\section{Patients and Methods}

Thirty-three patients with HT who underwent total thyroidectomy at Kuma Hospital (Kobe, Japan) between 1983 and 2006 were reviewed. According to the Guidelines of the Japanese Thyroid Society, all patients were diagnosed as having HT based on clinical findings: diffuse swelling of the thyroid gland without any other cause (such as Graves' disease) accompanied by any one of the following laboratory findings: (1) positive for antithyroid microsomal antibody or antithyroid peroxidase antibody, (2) positive for antithyroglobulin antibody, and (3) lymphocytic infiltration in the thyroid gland confirmed with cytological examination. Incidental findings of focal (nonspecific) lymphocytic thyroiditis in tumor-bearing thyroid tissue were excluded from this study. This study was approved by the Kuma Hospital Bioethical Committee.

Surgical specimens were fixed in formalin, routinely processed and embedded in paraffin. For light-microscopic examination, the sections were stained with hematoxylin-eosin (HE) and Victoria blue-HE stain.
Immunohistochemical studies were performed using automated Histofine Histostainer (Nichirei Bioscience Inc., Tokyo, Japan) according to the manufacturer's instructions.

The panel of antibodies included human immunoglobulin light chains (kappa and lambda; Dako A/S, Glostrup, Denmark), IgA (Dako), IgG (Dako), MCO011 (IgG4; Binding Site, Birmingham, UK), IgM (Dako) and a cocktail of 2G9 (CD21; Novocastra) and RB L25 (CD35; Novocastra). If necessary, polyclonal CD3 (Dako), 56C6 L26 (CD20; Dako), DFT-1 (CD43; Dako), 124 (bcl-2; Dako), AE1/3 (cytokeratin; Dako) and antithyroglobulin antibody (Dako) were also stained. Sections with known reactivity for the antibodies assayed served as positive controls and sections treated with normal rabbit and mouse serum served as negative controls.

In selected cases, genomic DNA was extracted from formalinfixed tissues after dewaxing of paraffin sections; then immunoglobulin heavy chain $(\mathrm{IgH})$ rearrangement was analyzed by polymerase chain reaction as described previously [11].

IgG4 HT was defined by the criteria of Li et al. [8, 9], i.e. (1) greater than 20 IgG4-positive plasma cells/high power field and (2) greater than $30 \%$ IgG4/IgG ratio. The degree of stromal fibrosis and lymphoid follicle formation was examined and expressed as $3+=$ severe, $2+=$ moderate, and $1+=$ mild.

Comparison of frequency data between the 2 groups was performed using the Fisher exact test. For continuous data, intergroup comparisons were performed using the Mann-Whitney $\mathrm{U}$ test.

\section{Results}

Fourteen cases were classified as IgG4 HT and the remaining 19 were classified as non-IgG4 HT.

\section{Clinical Findings}

Main clinical data of IgG4 HT and non-IgG4 HT patients analyzed in the study including age, gender, weight of resected thyroid gland, positivity for antithyroid gland antibodies, thyroid function and presence or absence of polyclonal hypergammaglobulinemia were collected and summarized in table 1 . There was a greater proportion of males in the IgG4 HT group, but the difference was not significant. In comparison with non-IgG4 HT, patients with IgG4 HT demonstrated a high incidence of hypothyroidism ( $\mathrm{p}<0.005)$.

\section{Pathological and Immunohistochemical Findings}

The main pathological findings are summarized in table 2 .

Histopathologically, both groups were characterized by follicular cell degeneration, dense lymphoplasmacytic infiltration and various degrees of lymphoid follicle formation and stromal fibrosis (fig.1a, b). Obstructive phlebitis was observed in both groups (IgG4 HT $=2$, non- 
Table 1. Summary of clinical findings in 33 cases

\begin{tabular}{lcc}
\hline & IgG4 HT & Non-IgG4 HT \\
\hline Number of cases & 14 & 19 \\
Gender (m:f) & $4: 10$ & $2: 17$ \\
Age $^{1}$, years & $41-66(54,56)$ & $44-66(56,54)$ \\
Weight of thyroid gland $^{1}$, g & $77-371$ & $65-367(190,203)$ \\
Thyroid test (+) $_{\text {Microsome test (+) }}$ & $8 / 12$ & $7 / 18$ \\
Hypothyroid & $11 / 12$ & $10 / 17$ \\
Hypergammaglobulinemia & & 9 \\
$\quad(>20 \%)$ & $8 / 9$ & $10 / 13$ \\
\hline
\end{tabular}

${ }^{1}$ Values represent mean range with the median given in parentheses.

IgG4 HT = 4) (fig. 1c). There were numerous mature plasma cells in a portion of the lymphoid follicles in 2 cases (IgG4 HT = 1, non-IgG4 HT = 1) (fig. 1d). Four cases (IgG4 HT = 4, non-IgG4-HT = 2) were diagnosed as fibrous thyroiditis because there was fibrous replacement of one third or more of the thyroid parenchyma $[4,5]$.

There was a greater proportion of fibrous thyroiditis in IgG4 HT. However, there was no significant difference between the 2 groups with regard to the stromal fibrosis (moderate/severe), lymphoid follicle formation (moderate/severe), or presence of obstructive phlebitis or fibrous thyroiditis.

An immunohistochemical study of the light chain determinant of intracytoplasmic immunoglobulins demonstrated a polytypic nature in the interfollicular area in all 33 lesions (kappa/lambda ratio approximately 3-4/1), whereas in the 2 lesions containing numerous plasma cells in the germinal center, intrafollicular plasma cells demonstrated marked kappa light chain predominance (kappa/lambda ratio was higher than 10/1) (fig. 2a, b). Intragerminal center plasma cells demonstrated a monotypic population based on the criteria of Lennert and Feller [12].

There were numerous IgG-positive plasma cells with scattered IgA- or IgM-positive plasma cells in all 33 cases. In 31 cases (IgG4 $\mathrm{HT}=13$, non-IgG4 HT = 1), IgG4- and/ or IgG-positive plasma cells were mainly located in the interfollicular area (fig. 2c, d) (interfollicular pattern), whereas in 2 lesions containing kappa-light-restricted plasma cells in the germinal centers, the majority of plasma cells in the germinal center as well as in the interfollicular area were also IgG4- and/or IgG-positive (fig. 2e)
Table 2. Summary of pathological findings in 33 cases

\begin{tabular}{lll}
\hline & IgG4 HT & Non-IgG4 HT \\
\hline Number of cases & 14 & 19 \\
Stromal fibrosis $(3 / 2 / 1)$ & $10 / 3 / 1$ & $8 / 4 / 7$ \\
Lymphoid follicles (3/2/1) & $1 / 10 / 3$ & $5 / 9 / 5$ \\
Obstructive phlebitis & 2 & 4 \\
Fibrous thyroiditis ${ }^{1}$ & 4 & 2 \\
$\begin{array}{l}\text { Presence of numerous plasma cells } \\
\quad \text { in germinal center }\end{array}$ & 2 & 1 \\
$\begin{array}{l}\text { Presence of numerous IgG4+ } \\
\quad \text { plasma cells in germinal center }\end{array}$ & 1 & 0 \\
\hline
\end{tabular}

${ }^{1}$ Fibrous replacement of one third or more of the thyroid parenchyma $[3,4]$.

(intrafollicular + interfollicular pattern). Thyroglobulin was detected in the cocktail of CD21 and CD35-antibody-positive follicular dendritic cell networks in the germinal centers as well as in the follicular epithelium in the cases examined (IgG4+ = 9, IgG4 =6) (fig. $2 \mathrm{f})$.

\section{Clinicopathological, Immunohistochemical and}

Genotypic Analysis of the Two Cases Showing an

Intrafollicular + Interfollicular Pattern

Unfortunately, information on staging was incomplete in the present series because the initial pathological diagnosis was HT. In both cases, adequate follow-up data were not available.

Histologically, there were no Dutcher bodies (intranuclear inclusions), centrocyte-like (CCL) cells or amyloid deposition. Staining for CD20 and CD3 showed the mixed nature of small lymphocytes. There were no CD20and CD43-positive CCL cells. There were not even lymphoepithelial lesions detected by immunostaining for cytokeratin in both lesions. Polymerase chain reaction analysis demonstrated that there was no clonal rearrangement of the immunoglobulin heavy chain.

\section{Discussion}

We demonstrated 2 distribution patterns of IgG4and/or IgG-positive plasma cells in IgG4 HT and nonIgG4 HT as well as in the lymph node lesion of IgG4-related sclerosing disease [10], i.e. the interfollicular pattern and the intrafollicular + interfollicular pattern. Interestingly, 1 case each of IgG4 HT and non-IgG4 HT showing 

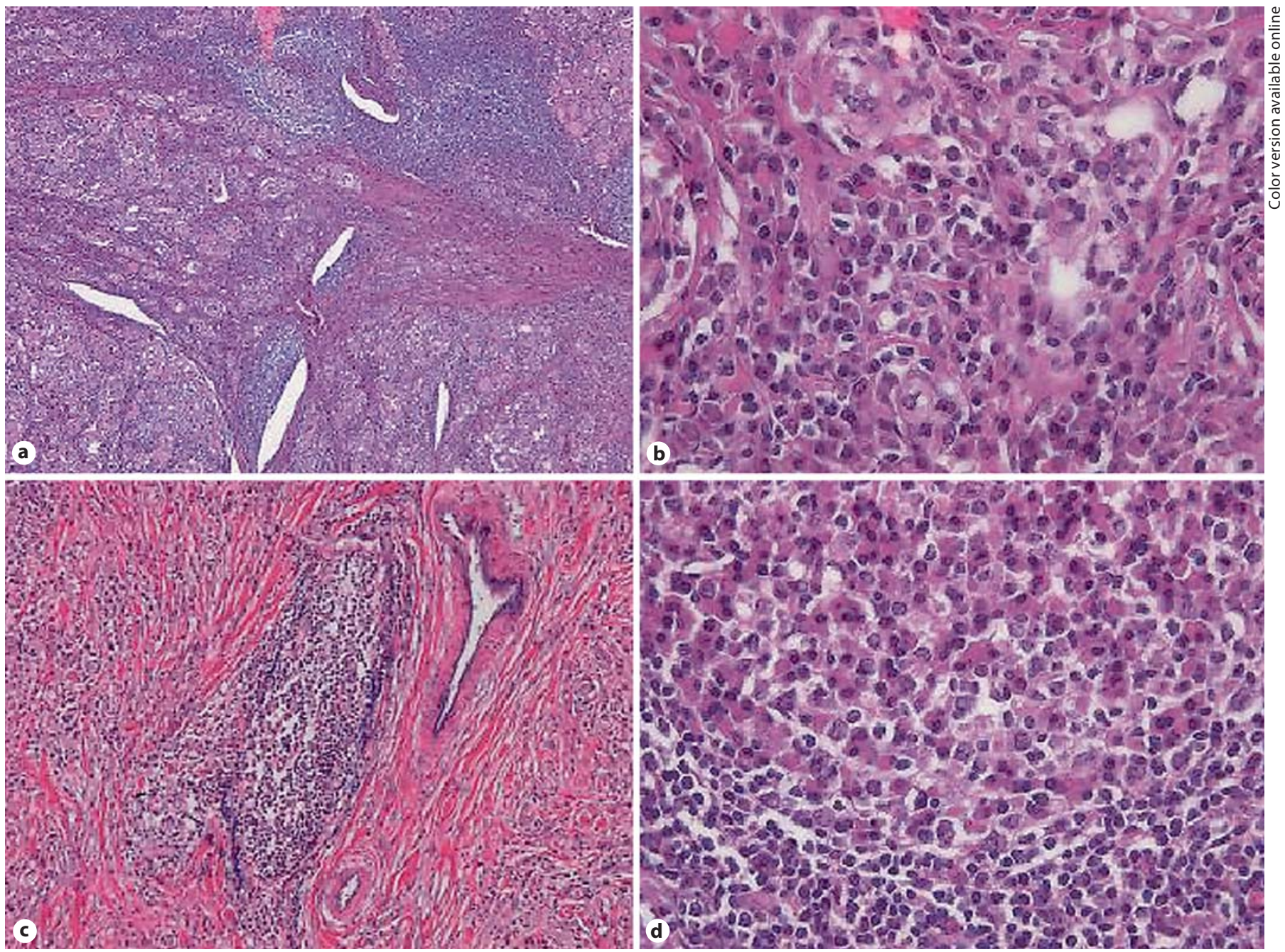

Fig. 1. a On a low-power field, the lesion was characterized by dense inflammatory processes, various degrees of lymphoid follicle formation and stromal fibrosis. HE. $\times 4$. b On a high-power field, there were prominent lymphoplasmacytic infiltrations associated with follicular cell degeneration. HE. $\times 40$. c On a medi-

um-power field, Victoria blue-HE stain demonstrated obstructive phlebitis. $\times 20$. d On a high-power field, numerous plasma cells were seen in the germinal center surrounded by mantle cells. HE. $\times 40$.

the intrafollicular + interfollicular pattern demonstrated kappa light chain-restricted germinal centers. These histological and immunohistological findings are similar to those of follicular colonization of extranodal marginal zone B cell lymphoma of the mucosa-associated lymphoid tissue (MALT) type showing prominent plasma cell differentiation [13]. In comparison with other types of MALT-type lymphoma, plasma cell differentiation is more prominent in thyroid MALT-type lymphoma [14]. Moreover, the majority of thyroid MALT-type lymphomas demonstrated an intracytoplasmic kappa light chain [15]. However, there were no CD20- and CD43-positive
CCL cells or lymphoepithelial lesions in either case [14, 16]. Using a cocktail of CD21 and CD35 antibodies, there was no broken follicular dendritic cell network, which is a characteristic immunohistochemical finding of follicular colonization of MALT-type lymphoma in either of the 2 lesions $[14,16]$. A few cases of IgG4-producing MALTtype lymphoma have been reported [17]. However, overall these immunohistochemical findings suggested that these 2 lesions differed from MALT-type lymphoma.

Riedel's thyroiditis has commonly been reported as a thyroid involvement of IgG4-related sclerosing disease by some researchers [18]. In this study, a small number 

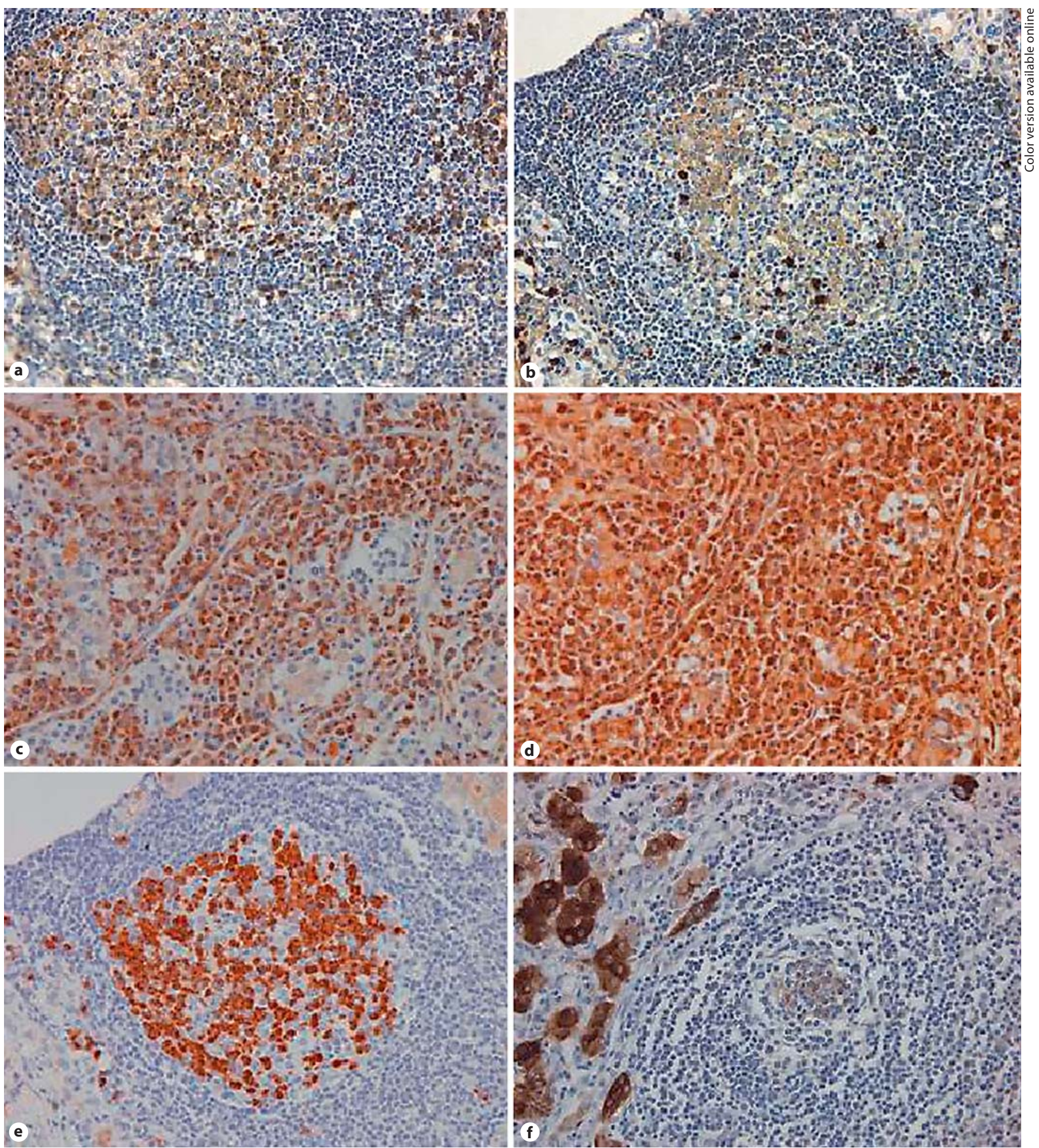

Fig. 2. In an immunohistochemical study, intrafollicular plasma cells demonstrated marked kappa light chain predominance (kappa/lambda ratio was greater than 10/1): kappa (a) and lambda (b). Same case as in figure 1c. $\times 20$. IgG4-positive plasma cells comprised more than $30 \%$ of IgG-positive plasma cells in the in-

terfollicular area: IgG4 (c) and IgG (d). $\times 20$. e Note numerous IgG4-positive plasma cells in the germinal center. $\times 20$. Same case as figure 1c. $\mathbf{f}$ Thyroglobulin was detected by a cocktail of CD21 and CD35 antibody-positive follicular dendritic cell networks in germinal centers as well as in follicular epithelium. $\times 10$. 
of IgG4 HT and non-IgG4 HT cases (IgG4 HT = 2, nonIgG4 HT = 4) demonstrated obstructive phlebitis which is a characteristic histological finding of Riedel's thyroiditis and IgG4-related sclerosing disease $[3-5,19]$. However, there was no extrathyroid fibrosis, which is another characteristic histological finding of Riedel's thyroiditis $[3,5]$. Thyroglobulin was detected at the follicular dendritic cell networks in germinal centers as well as in the follicular epithelium, which is seen in HT [20]. Furthermore, in a previous study, Harach and Williams [6] characterized plasma cell subsets in Riedel's thyroiditis by immunohistochemistry, demonstrating that IgA plasma cells but not IgG plasma cells were predominant in Riedel's thyroiditis. Therefore, as previously suggested by $\mathrm{Li}$ et al. $[8,9]$, we also considered that Riedel's thyroiditis is unrelated to IgG4-related sclerosing disease.
In conclusion, the present study demonstrated that the majority of cases with IgG4 HT and non-IgG HT showed an interfollicular distribution pattern of IgG4- and/or IgG-positive plasma cells. Moreover, a minority of IgG4 HT (7\%) and non-IgG4 HT contained IgG/kappa light chain-restricted germinal centers. However, the number of these cases is too limited to clarify the clinicopathological significance at present.

\section{Acknowledgments}

This work was supported by the Intractable Diseases, the Health and Labour Sciences Research Grants from Ministry of Health, Labor and Welfare.

\section{References}

1 Pearce EN, Farwell AP, Braverman LE: Thyroiditis. N Engl J Med 2003;348:2646-2655.

$\checkmark 2$ Hamano H, Kawa S, Horiuchi A, et al: High serum IgG4 concentrations in patients with sclerosing pancreatitis. N Engl J Med 2001; 344:732-738.

3 Masaki Y, Dong L, Kurose N, et al: Proposal for a new clinical entity. IgG4-positive multiorgan lymphoproliferative syndrome: analysis of 64 cases of IgG4-related disorders. Ann Rheum Dis 2009;68:1310-1325.

4 Katz SM, Vickery AL Jr: The fibrous variant of Hashimoto's thyroiditis. Hum Pathol 1974;2:161-170.

5 Volpe R: Etiology, pathogenesis, and clinical aspects of thyroiditis. Pathol Annu 1978;13: 399-413.

6 Harach HR, Williams ED: Fibrous thyroiditis. An immunopathological study. Histopathology 1983;7:739-751.

$\checkmark 7$ Komatsu K, Hamano H, Ochi Y, et al: High prevalence of hypothyroidism in patients with autoimmune pancreatitis. Dig Dis Sci 2005;50:1052-1057.

$>8$ Li Y, Bai Y, Liu Z, et al: Immunohistochemistry of IgG4 can help subclassify Hashimoto's autoimmune thyroiditis. Pathol Int 2009; 59:636-641.
-9 Li Y, Nishihara E, Hirokawa M, Taniguchi E, Miyauchi A, Kakudo K: Distinct clinical, serological, and sonographic characteristics of Hashimoto's thyroiditis based with and without IgG4-positive plasma cells. J Clin Endocrinol Metab 2010;95:1309-1317.

10 Sato Y, Kojima M, Takata K, et al: Systemic IgG4-related lymphadenopathy: a clinical and pathologic comparison to multicentric Castleman's disease. Mod Pathol 2009;22: 589-599.

11 Kuze T, Nakamura N, Hashimoto Y, Abe M: Most of CD30+ anaplastic large cell lymphoma of B cell type show a somatic mutation in the IgH V region genes. Leukemia 1998;12: 753-757.

12 Lennert K, Feller AC: Histopathology of Non-Hodgkin's Lymphomas (Based on the Updated Kiel Classification). Berlin, Springer, 1992.

13 Hussong JW, Perkins SL, Schnitzer B, Hargreaves H, Frizzera G: Extramedullary plasmacytoma. A form of marginal zone cell lymphoma? Am J Clin Pathol 1999;111:111115.

14 Isaacson PG, Norton AJ: Extranodal Lymphomas. Edinburgh, Churchill Livingstone, 1994.

15 Hyjek E, Isaacson PG: Primary B cell lymphoma of the thyroid and its relationship to Hashimoto's thyroiditis. Hum Pathol 1988; 19:1315-1326.
16 Isaacson PG, Chott A, Nakamura S, MüllerHermelink HK, Harris NL, Swerdlow SH: Extranodal marginal zone lymphoma of mucosa-associated lymphoid tissue (MALT lymphoma); in Swerdlow SH, Campo E, Harris NL, Jaffe ES, Pileri SA, Stein H, Thiele J, Vardiman JW (eds): WHO Classification of Tumours of Heamatopoietic and Lymphoid Tissues, ed 4. Lyon, IARC Press, 2008, pp 214-217.

17 Sato Y, Ohshima K, Ichimura K, et al: Ocular adnexal IgG4-related disease has uniform clinicopathology. Pathol Int 2008;58:465470 .

>18 Neild GH, Rodriguez-Justo M, Wall C, Connolly JO: 2006 Hyper-IgG4 disease: report and characterisation of a new disease. BMC Med 2006;4:23.

19 Kitagawa S, Zen Y, Harada K, et al: Abundant IgG4-positive plasma cell infiltration characterizes chronic sclerosing sialadenitis (Küttner's tumor). Am J Surg Pathol 2005;29: 783-791.

20 Kasajima T, Yamakawa M, Imai Y: Immunohistochemical study of intrathyroidal lymphoid follicles. Clin Immunol Immunopathol 1987;43:117-128. 